Noname manuscript No.

(will be inserted by the editor)

X.X. Yin • B. W.-H. Ng • K. Ramamohanarao •

A. Baghai-Wadji · D. Abbott

\title{
Exploiting Sparsity and Low-rank Structure for the Recovery of Multi-slice Breast MRIs with Reduced Sampling Error
}

Received: date / Accepted: date

\begin{abstract}
It has been shown that, magnetic resonance images (MRIs) with sparsity representation in a transformed domain, e.g. spatial finite-differences (FD), or discrete cosine transform (DCT), can be restored from undersampled $k$-space via applying current compressive sampling theory. The paper presents a model-based method for the restoration of MRIs. The reduced-order model, in which a full-system-response is projected onto a subspace of lower dimensionality, has been used to X.-X. Yin · B. W.-H. Ng · D. Abbott

Centre for Biomedical Engineering and School of Electrical $\&$ Electronic Engineering, The University of Adelaide, SA 5005, Australia

Tel.: $+61-8-8303-5748$

Fax: $+61-8-8303-4360$

E-mail: dabbott@eleceng.adelaide.edu.au

X.-X. Yin and K. Ramamohanarao

Department of Computer Science and Software Engineering, The Melbourne School of Engineering, The University of Melbourne, Victoria 3010, Australia

X.-X. Yin and A. Baghai-Wadji are with School of Electrical and Computer Engineering, Royal Melbourne Institute of Technology, Melbourne, Victoria 3000, Australia.
\end{abstract}

The MRI datasets were afforded by Q. Yang, Apollo Medical Imaging Technology Pty. Ltd. North Melbourne, VIC 3051, Australia and A. Pitman, Department of Anatomy and Cell Biology, The University of Melbourne and Sydney School of Medicine, The University of Notre Dame, Australia.

This work was supported in part by the Australian Research Council (ARC) Discovery Project funding scheme - Project No. DP0988064.

This project was also carried out under an Australian Research Council (ARC) Linkage Grant-LP0775463 
accelerate image reconstruction by reducing the size of the involved linear system. In this paper, the singular value threshold (SVT) technique is applied as a denoising scheme to reduce and select the model order of the inverse Fourier transform image, and to restore multi-slice breast MRIs that have been compressively sampled in $k$-space. The restored MRIs with SVT for denoising show reduced sampling errors compared to the direct MRI restoration methods via spatial FD, or DCT. Compressive sampling is a technique for finding sparse solutions to underdetermined linear systems. The sparsity that is implicit in MRIs is to explore the solution to MRI reconstruction after transform from significantly undersampled $k$-space. The challenge, however, is that, since some incoherent artifacts result from the random undersampling, noise-like interference is added to the image with sparse representation. These recovery algorithms in the literature are not capable of fully removing the artifacts. It is necessary to introduce a denoising procedure to improve the quality of image recovery. This paper applies a singular value threshold algorithm to reduce the model order of image basis functions, which allows further improvement of the quality of image reconstruction with removal of noise artifacts. The principle of the denoising scheme is to reconstruct the sparse MRI matrices optimally with a lower rank via selecting smaller number of dominant singular values. The singular value threshold algorithm is performed by minimising the nuclear norm of difference between the sampled image and the recovered image. It has been illustrated that this algorithm improves the ability of previous image reconstruction algorithms to remove noise artifacts while significantly improving the quality of MRI recovery.

Keywords singular value threshold $\cdot$ model order reduction $\cdot$ sparse sensing $\cdot$ MRI $\cdot k$-space $\cdot$ low rank matrix $\cdot$ finite differences $\cdot$ discrete cosine transform

\section{Introduction}

Compressive sensing has been proven to be an important technique for speedy measurements in many MRI applications [1]. The challenge is to reduce the amount of acquired data without degrading the image quality, since direct reconstructions of signals undersampled in $k$-space exhibit aliasing artifacts. Many recent proposed methods for reduced data imaging allow recovery of an image with sparse representations, but these typically show some aliasing artifacts which lie within the imposed data fidelity constraints. This paper adopts a singular value threshold (SVT) to reduce and select the model order (number of image basis functions) for the recovery of multi-slice breast MRIs. Here, we use a denoising scheme of compressively sampled $k$-space measurements in combination with known image reconstruction algorithms, i.e. image reconstruction illustrated in [1], which leads to reduced errors in the recovered image. The advantage of the current model order reduction and selection (MORS) scheme is the use of compact mathematical modeling to analyze an ensemble of MRI matrices using singular value decomposition with a certain threshold. It is observed that the smaller numbers of singular values used allows improved recovery of MRIs, where the image boundaries are shown to be clearer with reduced noise artifacts and the recovered image intensities 
are considered to be more accurate than the MRIs that are reconstructed without the MORS scheme being used. Under this scheme, a tradeoff exists between relative error and recovery rates. The relative error rate compares the images reconstructed using both the approximated and the exact values to the original image, while the recovery rate measures the reconstruction error (image pixels located) outside the region of interest between the recovered (approximated) and exact image to the original. The algorithm is computationally efficient in the removal of noisy pixels since it employs matrices with low rank.

In contrast to the recent method [1] that directly selects significant image pixels from some sparsifying transform, e.g. finite differences (FD), discrete cosine (DCT) or wavelet transform (DWT), this paper selects significant pixels from MRIs after transformation from undersampled $k$-space. The selection strategy is to compare the reconstructed MRI (from undersampled $k$-space) with a Wiener filtered (smoothed) version of itself, then choosing as significant the pixels where the difference does not exceed some pre-determined threshold. This method improves the degree of automation in the overall processing scheme. It is observed that singular value (SV) thresholding of significance-selected image improves the quality of the final image obtained by the final FD-based compressive sampling recovery. By carefully choosing the singular value threshold (and thus the spectrum norm), we are able to control the relative error rate of sampled matrices and the relative recovery rate of an image. For multi-slice MRIs with image size of $260 \times 260 \times 5$ (the data set used in this paper for illustration purposes), we can set a sample rate of $94 \%$ after SVT and a desired the relative error of at most $1.7 \%$, the average recovery rate can reach over $98 \%$. The peak signal-to-noise ratio (SNR) with application of the SVT for denoising is 12 , which is a significant improvement to the values of 2 for FD via identity transform (IT) and 3 for FD via DCT [1].

Sections II and III in this paper represent the methodology and source data acquisition that are used to perform the breast contrast MRI measurements and relevant image processing. Section IV presents resultant images and discusses the implementation of these methods for analysis purposes. Section V concludes the paper.

\section{Method}

The proposed method contains three parts: (i) Undersampled MRI acquisition, (ii) denoising and (iii) FD-based recovery. For part (i), the undersampling of the MRI measurement in $k$-space is solely determined by the MRI machine in practice. This part is modelled in this paper as an undersampled $2 \mathrm{D}$ Fourier transform on the ground truth $\mathrm{MRI} \mathbb{X}(x, y)$. After applying a $2 \mathrm{D}$ inverse Fourier transform (IFT), we achieve a transformed image with incoherent artifacts, $\mathfrak{A}(x, y)$. Part (ii) is our proposed denoising scheme aimed at reducing the noise in reconstructed image. The denoising scheme consists of applying a Wiener filter $g(x, y)$ to the image $\mathfrak{A}(x, y)$ and computing the difference between the unfiltered and filtered images to produce $\Delta n(x, y)$. These differences are then thresholded using a pre-determined value $\epsilon$ and pixels in $\mathfrak{A}(x, y)$ that corresponds to locations where $\Delta n(x, y)<\epsilon$ are 
retained with the remainder discarded, producing $\mathbf{X}(x, y)$. A singular value decomposition of $\mathbf{X}(x, y)$ is computed and then only the singular values exceeding some threshold are retained, producing the singular value thresholded (SVT) image $\mathbf{M}(x, y)$. In part (iii), the image $\mathbf{M}(x, y)$ is applied to a FD-based compressive sensing algorithm to produce the final reconstructed MRI $\mu(x, y)[1]$. A block diagram for the procedure is provided in Fig. 1.

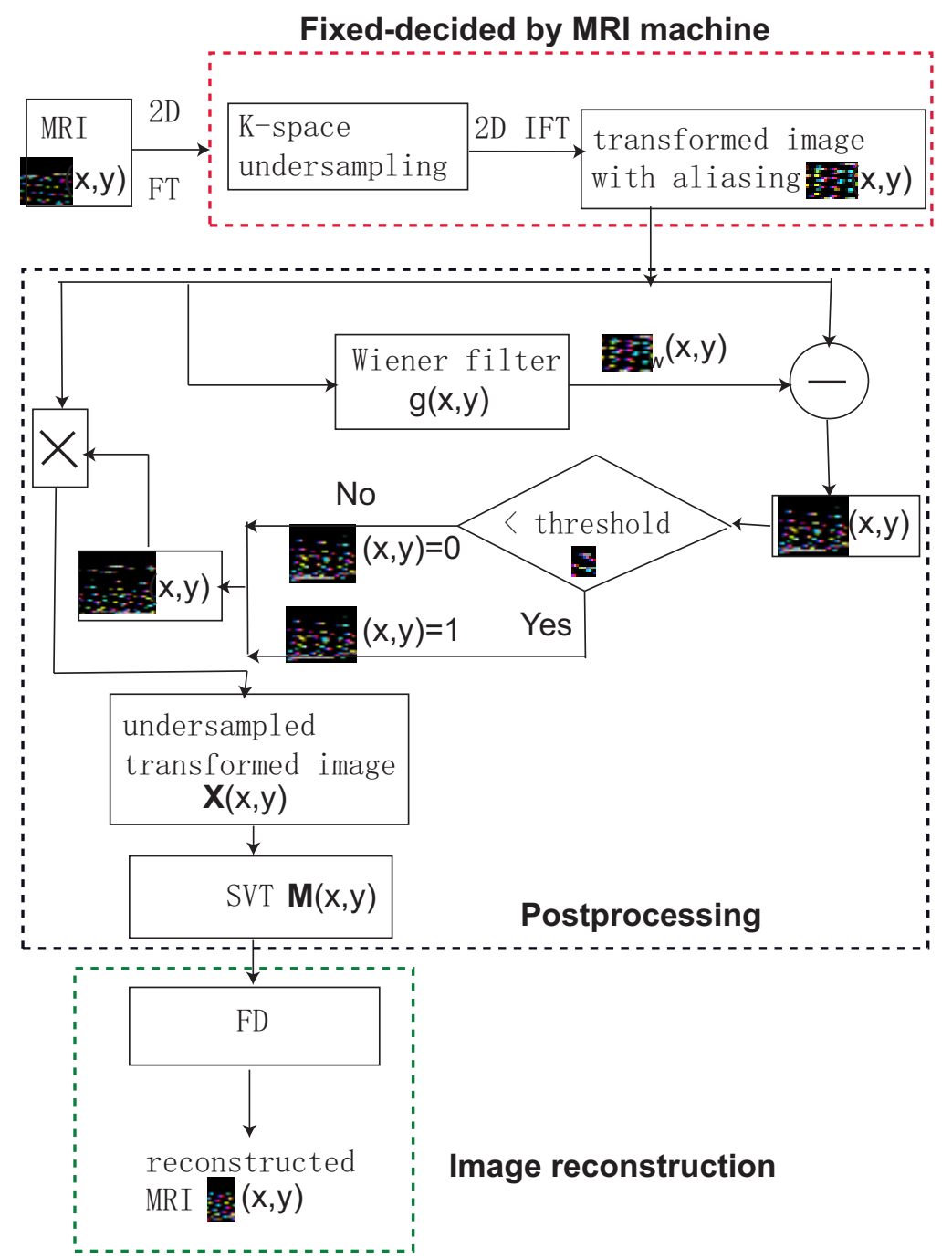

Fig. 1 This is to illustrate the algorithm regarding compressively sampled MRI reconstruction with application of SVT as a denoising operation.

\subsection{The K-Space presentation}

$k$-space is a common term in MRI physics which refers to the spatial (either 2-D or 3-D) frequency domain in wider engineering literature. The $k$-space presentation is a method that is widely used 
in MRI, and was independently introduced in 1983 by Ljunggren [2] and Twieg [3]. Its complex values are sampled during an MR measurement, in a predetermined scheme controlled by a pulse sequence, i.e. an accurately timed sequence of radio frequency and gradient pulses. In practice, $k$ space often refers to the temporary image space, usually a matrix, in which data from digitized MR signals are stored during data acquisition. When $k$-space is full (at the end of the scan) the data are processed to produce a final image. From a processing perspective, $k$-space holds raw data before any reconstruction computations.

It is desirable to reduce the signal acquisition time in MRI and undersampling $k$-space is a technique for achieving this. An undesirable side-effect of this strategy is the appearance of artifacts in the recovered images, although compressive sensing (CS) techniques can be used to reduce this; our algorithm is based on the broad principles of CS. Considering the aliasing appears incoherent when undersampling in $k$-space, it is possible to use variable-density sampling schemes for Cartesian coordinate system to widely distribute the artifacts and reduce their visual impact. In such schemes, high energy low-frequency image components alias less than lower energy higher frequency components, and the interference appears as white noise in the image domain. The undersampling method is carried out by sampling less near the $k$-space and more in the periphery of $k$-space and the number of $k$-space samples should be roughly two to five times the number of sparse coefficients [1, 4]. In this paper, we select a number of entries for illustration purposes, and the image that is transferred from undersampled $k$-space with such number of entries shows obvious aliasing artifacts, illustrated in Fig. 3(a) and Fig. 4(b), and can be used to compare the image reconstruction quality according to the proposed algorithm in this paper and methods suggested in the literature.

\subsection{Wiener filtering}

There are a number of methods to assess and remove image noise, i.e. noise classification [5, 6], and noise filtering [7]. Noise can be reduced certainly by a low-pass filter or smoothing operation without affecting the quality of the rest of the image. The noise pixels are believed to show sharp variety. The pixel with variety in intensity larger than certain value according to the reference smoothed image, will be viewed as being interrupted by noise. This is the principle behind the popular median filtering technique for noise removal [? ]. Evaluation for noise pixels of MRI is beyond the scope of this paper. Instead, we propose to use Wiener filtering to assist pre-selection of image pixels during denoising operation. The Wiener filter was proposed by Norbert Wiener in 1949 [7] and it is based on statistical approach towards estimating additive noise present in a signal.

Assume the image $\mathfrak{A}(x, y)$ to be modelled as the true image corrupted by incoherent noise $n(x, y)$ caused by undersampled $k$-space measurements, i.e. we have $\mathfrak{A}(x, y)=S(x, y)+n(x, y)$, with $S(x, y)$ the desired noiseless signal. In order to minimise the influence of the noise $n(x, y)$, we use a Wiener filter with impulse response $g(x, y)$, so that

$$
S(x, y)+\tilde{n}(x, y)=g(x, y) \otimes \mathfrak{A}(x, y)
$$


such that

$$
\|\tilde{n}(x, y)\|^{2} \ll\|n(x, y)\|^{2}
$$

resulting in

$$
\mathfrak{A}_{w}(x, y)=\hat{S}(x, y)=S(x, y)+\tilde{n}(x, y)
$$

where $\mathfrak{A}_{w}(x, y)=\hat{S}(x, y)$ is the estimated Wiener smoothed image, and $\|x\|^{2}$ is Euclidean norm. The sparsity of the image $\mathbf{X}$ is obtained by selecting the significant pixels with intensity in between the images $\mathfrak{A}(x, y)$ and $\mathfrak{A}_{w}(x, y)$ that should be within a threshold. That is

$$
\begin{aligned}
\Delta n(x, y) & =\mathfrak{A}_{w}(x, y)-\mathfrak{A}(x, y), \\
\text { and } & \widetilde{\Delta n}(x, y)= \begin{cases}0, & \text { if } \Delta n(x, y)>\epsilon \\
1, & \text { if } \Delta n(x, y) \leq \epsilon\end{cases}
\end{aligned}
$$

The sparse matrix $\mathbf{X}$ is the elementwise product of $\widetilde{\Delta n}$ and $\mathfrak{A}$.

\subsection{Postprocessing of MRIs with singular value thresholding}

The singular value thresholding algorithm is used in this paper as an effective denoising scheme of MRIs for accurate image reconstruction. It is introduced by [8], which applies a soft-thresholding rule to the singular values of sparse representation of image matrices in $\mathbb{R}^{n_{1} \times n_{2}}$ with rank of $r$.

Consider a set of MR measurements $\bar{\gamma}\left(k_{x}, k_{y}\right)$, sampled at locations in $k$-space $\Phi=\left\{\left(k_{x_{i}}, k_{y_{i}}\right), i=\right.$ $1,2, \cdots l\}$, with undersampling produced by $l<N$ where $N=n_{1} \times n_{2}$ is the total number of pixels in the image. Direct reconstruction from this set of undersampled $k$-space measurements produce $\gamma(x, y)$, where $(x, y)$ is the spatial location coordinate, can be rewritten in vector form as $\gamma(x, y)=F^{*} \bar{\gamma}\left(k_{x}, k_{y}\right)$, where, $F^{*}$ is the inverse Fourier transform operator. If we re-arrange the signal intensity of MRIs $\gamma(x, y)$ in a matrix form

$$
\mathfrak{A}=\left[\begin{array}{ccc}
\gamma\left(x_{1}, y_{1}\right) & \cdots & \gamma\left(x_{1}, y_{n_{2}}\right) \\
\vdots & & \vdots \\
\gamma\left(x_{n_{1}}, y_{1}\right) & \cdots & \gamma\left(x_{n_{1}}, y_{n_{2}}\right)
\end{array}\right]
$$

The matrix $\mathfrak{A}$ is then processed as discussed in the previous section to produce $\mathbf{X}$. For a typical image with high degree of spatial correlation, the rows and columns of $n_{1} \times n_{2}$ matrix are linearly dependent, the rank of $\mathbf{X}$ is given by $r<\min \left(n_{1}, n_{2}\right)$. An arbitrary $n_{1} \times n_{2}$ matrix of rank $r$ can be written in terms of its singular value decomposition (SVD):

$$
\mathbf{X}=\mathbf{U} \Delta \mathbf{V}^{H} .
$$

Here, $H$ stands for transposition. This decomposition implies that the image signal intensity $\gamma(x, y)$ can be expressed as a weighted linear combination of $r$ image basis functions [9]:

$$
\gamma(x, y)=\sum_{i=0}^{r-1} \eta_{i}(x) v_{i}(y)=\sum_{i=0}^{r-1} u_{i}(x) \varpi_{i}(y) .
$$


The image basis functions $\eta_{i}(x)$ and $\varpi_{i}(y)$ are the $1 \times n_{2}$ row vectors and $n_{1} \times 1$ column vectors of $\mathbf{U} \Delta$ and $\Delta \mathbf{V}^{H}$ in Eq. (7), respectively, while the spatial weights $u_{i}$ and $v_{i}$ correspond to the rows and columns of the matrix $\mathbf{U}$ and $\mathbf{V}$, respectively.

In this paper, we use the following strategy to reduce and select model orders of image basis functions, and to reconstruct MRIs. Firstly, we estimate the weight vectors $u_{i}(x)$ or $v_{i}(y)$ with $i=0,1, \cdots, r-1$ using the SVD of the training image voxels' signal intensity series. The training data consists of Fourier reconstructed image data from undersampled measurements in $k$-space. The image basis functions $\eta_{i}(x)$ or $\varpi_{i}(y)$ with $i=0,1, \cdots, r-1$ from the undersampled training image are also obtained from the SVD. The model order, or the effective number of image basis functions retained in the SVD, is determined by a threshold for singular values. Define the soft-thresholding operator $\mathbb{S}_{\tau}$ by:

$$
\mathbb{S}_{\tau}(\mathbf{X})=\mathbf{U} \mathcal{S}_{\tau}(\Delta) \mathbf{V}^{H} ; \quad \mathcal{S}_{\tau}(\Delta)=\operatorname{diag}\left(\left\{\max \left(\alpha_{i}-\tau, 0\right)\right\}\right)
$$

where $\mathbf{U}$ and $\mathbf{V}$ are, respectively, $n_{1} \times r$ and $n_{2} \times r$ matrices with orthogonal columns, and $\alpha_{i}$ is the $i$-th largest singular value $(\mathrm{SV})$ of matrix $\mathbf{X}$, all of which consist of a diagonal matrix $\Delta=\operatorname{diag}\left\{\alpha_{i}\right\}_{1 \leq i \leq r}$.

For each threshold $\tau \geq 0$ and restored matrix $\mathbf{M} \in \mathbb{R}^{n_{1} \times n_{2}}$, the SVT operator is approximately equivalent to the solution obtained from the optimization problem: $\mathbb{S}_{\tau}(\mathbf{M})=\operatorname{argmin} \mathbf{X} \frac{1}{2}\|\mathbf{X}-\mathbf{M}\|^{2}+$ $\tau\|\mathbf{X}\|_{*}$, where $\|X\|_{*}$ is a set of nuclear norm, and $\|\mathbf{X}\|^{2}$ is the Euclidean norm. The nuclear norm is also known as trace norm [10]; if we denote the singular values using $\sigma_{i}$, the nuclear norm of $A$ is defined on the space of $n_{1} \times n_{2}$ matrices by

$$
\|A\|_{*}=\operatorname{Tr}\left(\sqrt{A^{*} A}\right)=\sum_{i=1}^{\min \left\{n_{1}, n_{2}\right\}} \sigma_{i} .
$$

We use linearized Bregman iterations [11] to calculate soft-thresholding singular values recursively. We fix $\tau>0$ and a sequence $\delta_{k}$ label positive step sizes. For the current MRI application, let $\mathbf{Y}^{k}, k=0,1, \cdots$ be a sequence of images, with $\mathbf{Y}^{0}$ being the original image $\mathbf{X}$ obtained from earlier processing. In each iteration, the largest SVs of $\mathbf{Y}^{k}$ are calculated, and the number of them is incremented with iterations, with the only first largest singular value considered in the first iteration. The matrices $\mathbf{Y}^{k}$ and $\mathbf{M}^{k}$ are inductively defined for $k=1,2, \cdots$ by

$$
\begin{aligned}
\mathbf{M}^{k} & =\mathbb{S}_{\tau}\left(\mathbf{Y}^{k-1}\right), \\
\mathbf{Y}^{k} & =\mathbf{Y}^{k-1}+\delta_{k} G_{\omega}\left(\mathbf{X}-\mathbf{M}^{k}\right) .
\end{aligned}
$$

If the relative error rate, defined by $\|\mathbf{X}-\mathbf{M}\|_{*} /\|\mathbf{X}\|$, is small enough, then the iteration is stopped. We define $\omega$ as the region of interest (ROI) and $|\omega|$ as the number of samples in the ROI. The expression, $1-\left\|G_{\omega}(\mathbf{X}-\mathbf{M})\right\|_{*} /\left\|G_{\omega}(\mathbf{M})\right\|_{*}$, is called the relative recovery, which quantifies the reconstruction quality of an image. We use $G_{\omega}$ to denote the orthogonal projector onto the span of matrices vanishing outside of $\omega$ so that the $(i, j)$-th component of $G_{\omega}(\mathbf{X})$ is equal to $X(i, j) \in \omega$ and zero otherwise. It is evident that, for each $k \geq 1, \mathbf{Y}^{k}$ vanishes outside of $\omega$, and is therefore sparse, a fact which can be used to speed up computations. The matrices $\mathbf{M}^{k}$ turn out to have low rank, and 
hence the algorithm has a minimum storage requirement as we only need to keep principal factors in memory. Lanczos iteration [12] is used to find largest singular values of a matrix during each iteration mentioned above. It transforms the given matrix into a tridiagonal matrix, which is real and symmetric. It uses the Gram-Schmidt process [13] to reorthogonalize them into a basis spanning the Krylov subspace $[14,15]$ corresponding to the given matrix.

From the section of 'Method', $\mathfrak{A}$ is a transformed image from undersampled $k$-space data. We use $\psi$ to denote the linear operator that transforms the image to a sparse representation. In this paper, this is implemented by computing the difference between the (noisy) image $\mathfrak{A}$ and its smoothed version, called the reference image in this context, which is obtained by Wiener filtering. The pixels in the sparse image representation correspond to those locations where the difference does not exceed a threshold. The sparse representation of image matrix $\mathbf{X}=\psi \mathfrak{A}$, is finally recovered to the image matrix $\mathbf{M}$ using SVT method described above. From our experiments, the obtained images $\mathbf{M}$ show the ability of SVT to remove the incoherent aliasing artifacts resulting from undersample of $k$-space.

\subsection{Reconstruction of compressed sensing MRI}

According to the recently developed theory of compressive sensing (CS), images with sparse representations can be recovered from randomly undersampled $k$-space data, if an appropriate nonlinear recovery scheme is carried out. The sparsity of the image is the percentage of sparsifying transform coefficients sufficient for image reconstruction. Artifacts due to random undersampling cause aliasing interference in the transformed image, where the significant coefficients are believed to stand out above the interference. A non-linear thresholding scheme, called finite-differences (FD) method, is used as one of several sparsifying transformations in MRI recovery [1]. The finite-differences reconstruction is performed by minimising the $l_{1}$ norm of a sparse transformed image with an additional penalty proportional to the total variation (TV) [16] in an image. This method is defined as

$$
\text { minimise }\|\hat{\psi} \mu\|_{1}+\delta \Upsilon(\mu) \quad \text { s.t. }\left\|F_{u} \mu-\zeta\right\|_{2}<\epsilon
$$

where $\mu$ labels the recovered image vector of interest, with the initial input image being subject to the denoised image $\mathbf{M}$ described in Sec $2.3 ; \hat{\psi}$ denotes the linear sparse operator; $F_{u} \mu$ is the undersampled Fourier transform of image vectors, $\zeta$ is the measured $k$-space data from scanner, in our case, it is undersampled Fourier transform of original image $\mathbb{X} ; \epsilon$ controls the fidelity of the reconstruction to the measured data, and $\delta$ is a parameter to control the effect of the penalty term. In our paper, $\delta$ is selected as 0.01 . The value is empirically determined and it has been proved to work well for our data sets. The $l_{1}$ norm is defined as $\|x\|_{1}=\sum_{i}\left|x_{i}\right|$, and $\Upsilon(\mu)$ is defined on an interval $[a, b]$ as $Y_{b}^{a}(\mu)=\sup _{P} \sum_{i=0}^{n_{P}-1}\left|\mu\left(x_{i+1}\right)-\mu\left(x_{i}\right)\right|$, where the supremum runs over the set of all partitions $\mathcal{P}=\left\{P=\left\{x_{0}, \cdots, x_{n_{P}}\right\}\right\}$ with $P$, a partition of the given interval $[a, b][16]$. The constraint $\left\|F_{u} \mu-\zeta\right\|_{2}<\epsilon$ enforces data consistency. It should be emphasized that the recovered image $\mu$, as discussed in the section of 'Method', is treated in our algorithm as a denoised image $\mathbf{M}$ that has been restored after 
sparse transform, so as to reduce the aliasing artifacts. The sparse operator $\hat{\psi}$ can be performed via DCT or identity transform although the latter is generally used as it requires no computation and thus has fast running times in combination with the SVT for denoising.

\subsection{Peak signal-to-noise ratio}

The phrase 'peak signal-to-noise ratio' is often abbreviated to PSNR. It is most commonly used to measure quality of reconstruction for lossy compression. It is formed by calculating the ratio between the maximum possible power of a signal and the power of corrupting noise that affects the fidelity of its representation. The signal in this case is the original data, and the noise is the error introduced by compression [17]. Because many signals have a very wide dynamic range, PSNR is usually expressed in terms of the logarithmic decibel scale. Mean Square Error (MSE) is another parameter used to easily define PSNR. For two $m \times n$ monochrome images $\mathbf{I}$ and $\mathbf{K}$ where one of the images is considered to be a noisy approximation of the other, MSE is defined by:

$$
\mathrm{MSE}=\frac{1}{m n} \sum_{i=0}^{m-1} \sum_{j=0}^{n-1}[I(i, j)-K(i, j)]^{2} .
$$

The PSNR is defined by:

$$
\mathrm{PSNR}=10 \cdot \log _{10}\left(\frac{\mathrm{MAX}_{I}^{2}}{\mathrm{MSE}}\right)=20 \cdot \log _{10}\left(\frac{\mathrm{MAX}_{I}}{\sqrt{\mathrm{MSE}}}\right)
$$

Here, MAX $_{I}$ is the maximum possible pixel value of the image that is restored after sparse transformation. Obviously, a higher value of PSNR is preferable because it implies that the ratio of signal to noise is higher.

\subsection{Source data}

DCE-MRI may be especially valuable for screening patients at high risk of developing breast diseases. The current work that studies the reconstruction of breast MRIs uses the datasets according to first time slice of DCE-MRI. The clinical dynamically contrast enhanced breast MRI examination is performed on a $1.5 \mathrm{~T}$ clinical magnet (Magnetom Avanto, Siemens, Erlangen, Germany). The entire MR examination is securely de-identified and not re-identifiable before being transferred from the acquisition workstation. The examination includes a dynamic contrast enhanced axial T1 weighted (T1W) sequence (parameters below). The baseline dataset from this sequence (before contrast injection) and dataset at 60 seconds post contrast injection are used in this study. Table 1 shows MRI acquisition parameters for the Siemens 1.5T Avanto magnet. Dynamic contrast enhanced axial gradient echo T1 weighted sequence is repeated six times: once before contrast administration, and five times after contrast administration (at 60 second intervals). The contrast, composed of $0.16 \mathrm{mmol} / \mathrm{kg}$ of gadopentetate dimeglumine (Magnevist, Schering AG, Berlin, Germany) is injected intravenously. The relevant parameter configuration is illustrated in Table 1. 
Table 1 MRI acquisition parameters for the Siemens 1.5T Avanto magnet. The asterisk denotes the parallel imaging technique employed, GRAPPA (Generalized Autocalibrating Partially Parallel Acquisition) with acceleration factor equal to 2 .

\begin{tabular}{cc}
\hline Sequence & GRE $(\text { VIEWS })^{*}$ \\
Image mode & $3 \mathrm{D}$ \\
Repeated time $(\mathrm{ms})$ & 5.11 \\
Echo time $(\mathrm{ms})$ & 2.71 \\
\hline Inverse time $(\mathrm{ms})$ & - \\
\hline Flip angle $(\mathrm{deg})$ & 15 \\
\hline Field of view (FOV) $(\mathrm{mm})$ & 340 \\
\hline Acquisition plane & Axial \\
\hline Slice mm/gap mm & $1.0 / 0.0$ \\
\hline No. of slice & $160 /$ slab \\
\hline Matrix freq/phase & $448 \times 448$ \\
\hline Pixel mm in plane & $1.10 \times 0.80$ \\
No. of signals acquisition & 1 \\
Fat suppression & Frequency selective \\
Repeats before contrast & 1 \\
Repeats after contrast & 5 \\
Repetition time $(\mathrm{s})$ & 60 \\
Total time (min) & $6: 24$ \\
\hline
\end{tabular}

\section{Results}

This section illustrates the resultant images after applying the algorithms represented above. The MRI, for convenience, has been cut to image size to $260 \times 260 \times 30$. We only apply the algorithms represented on 26-30 layers for verifying the feasibility of our approach. The number of undersampled $k$-space datasets is 22,273 out of $260 \times 260=67,600$ Fourier samples $(33 \%)$. The number of image pixels for the sparse representation of the current MRIs is equal to $94 \%$ of the image size (number of non-zero pixels in $\mathbf{M}$ ). The number of pixels to be selected is sufficient to allow a clear and quality reconstruction via SVT. The certain threshold is $13 \times n(n=260)$ for the calculation of largest singular values to achieve the image presentation, illustrated in Fig. 2 and Fig. 4(b). In these cases, we select the pixels with variety in intensity between smoothing and aliased images smaller than 40 as image pixels for denoising. This is to achieve $94 \%$ of the image size for sparse image representation mentioned before. These MRIs are reconstructed at layer 26.

Fig. 2(a) illustrates original breast MRI data at the 26th layer. Fig. 3(a) illustrates the transform MRI from the compressively sampled $k$-space domain, which produces an incoherent aliasing effect compared to Fig. 2(a); Fig. 3(b) illustrates Wiener filtered smoothed image, and Fig. 3(c) is for MRI with sparse representation, the number of data entries of which is 63,877 out of 67,600 , original image size on this layer. The black dots shown in this image indicate the missing pixels. We want to highlight that though the preselected image size only saves $6 \%$ of original image, the image matrix 
rank is reduced with obvious reduced number of singular values, which means the further reduced computational complexity. It shows good image recovery quality and reduced data storage space during the image processing procedure compared with the sparse image recovery with direct use of the finite difference operation and discrete cosine transform. For comparison, we also illustrate $85 \%$ image entries for MRI restoration.

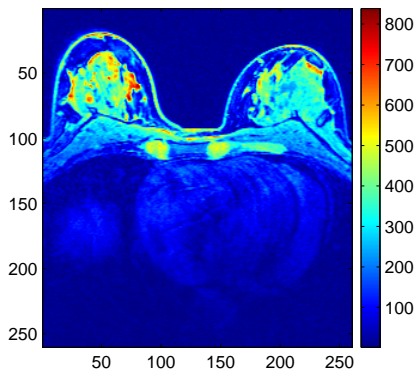

(a)

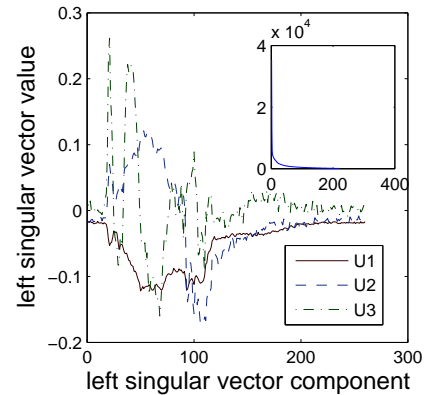

(b)

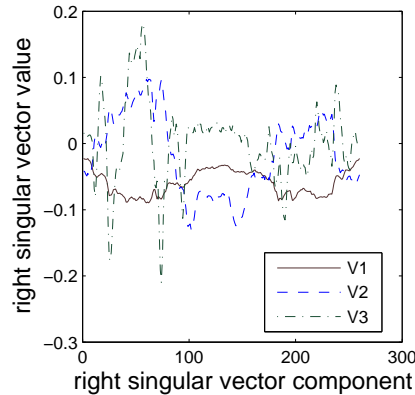

(c)

Fig. 2 Illustrations of at the layer of 26, (a) the original measurement breast MRI data. (b) and (c) Illustrations of, under $94 \%$ of all samples that are obtained, the decomposed left- and right singular vectors corresponding to the first (red line), second (blue line), third (green line) largest SVs. The insertion in (b) shows first several largest singular values of the image recovery matrix. It is observed that the vibrating frequency of each of singular vector is increased with reduced singular value that each of the singular vector corresponds to.

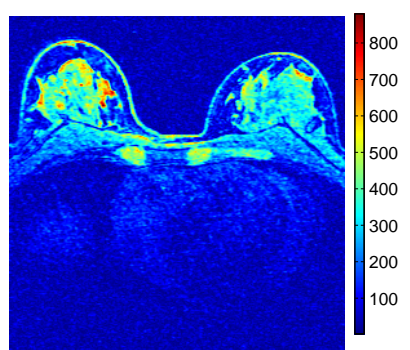

(a)

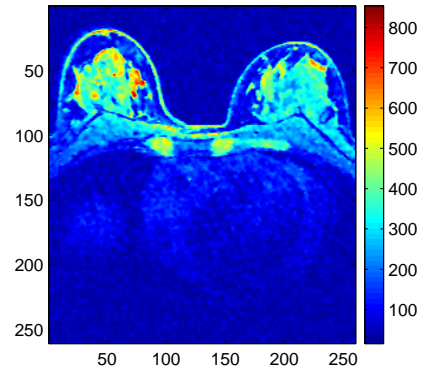

(b)

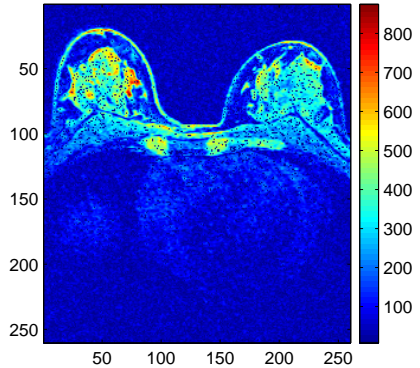

(c)

Fig. 3 Illustrations of, at the 26th layer, (a) sparse transform data from compressively sampled $k$-space domain, (b) Wiener filtered smoothed image, and (c) final MRI with sparse representation, the black dots shown on the image are the missing pixels. 
For further evaluating the effect of Wiener smoothing, we subtract the smoothed MRI and the transformed MRI from the original MRIs, then we separate the resultant object images with verity in intensity larger than zero from background. Fig. 4(a) and (b) illustrate the resultant segmented images in terms of the smoothed image in Fig. 3(a) and the MRI after being transformed in Fig. 3(b). It is observed that the segmented smoothed image illustrated in Fig. 4(a), shows nearly uniform increment in intensity in the object region, without introducing extra noise pixels, while the segmented transformed image in Fig. 4(b) is noisy with noise pixels located in the whole background region. This are aliasing artifacts after being transformed from undersampled K-sapce domain. If we compare the smoothed image with the transformed image, then the missing pixels are determined according to the difference in the reconstructed intensity that is large enough. These missing pixels are considered as to be related to the noise pixels represented in Fig. 3(b).

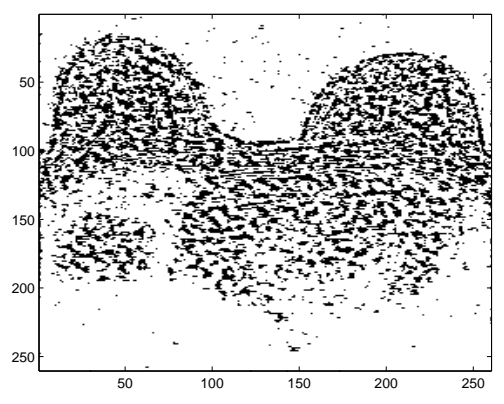

(a)

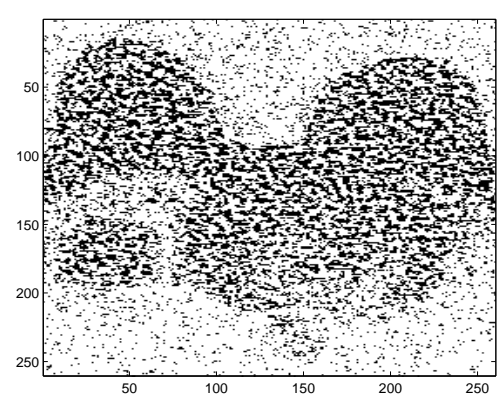

(b)

Fig. 4 Illustrations of the segmented images, in terms of intensity variation, for (a) Wiener smoothing of an image and (b) transformed image from sampled $k$-space in comparison with original measurement. The transformed image segment shows obvious noise pixels in the background region.

Fig. 5(a) shows the reconstructed image with sparsity. It is realised via inputting the SVT based denoising data to finite difference reconstruction with application of an identity transform. The method takes 212 seconds to perform the SVT based denoising operation and 50 seconds for the identity transform (IT) based finite difference (FD) operation. The peak signal to noise ratio (PSNR) is 12. Fig. 5(b) shows the sole finite difference reconstruction, the elapsed time of which is 42 second. It is obvious to see some aliasing effect from finite difference reconstructed image compared to SVT denoised image, the PSNR of which equals to 2 though the elapsed time is slightly reduced. Fig. 5(c) plots the difference in intensity according to the data along 100th row of the reconstructed image and original measured MRI. The red line is the plot in regards to identity reconstruction, which shows obvious error in reconstructed intensity compared to the original measured data. The green dash 
dot line in terms of SVT based denoising scheme shows smaller amount of error in reconstructed intensity than IT.

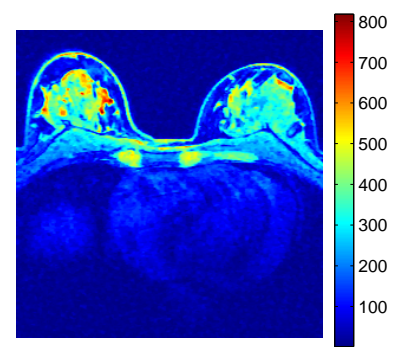

(a)

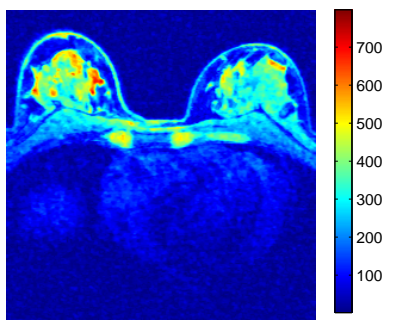

(b)

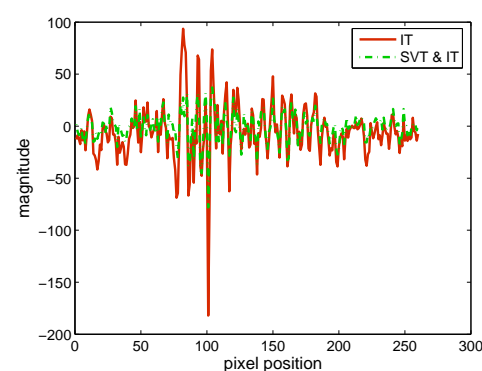

(c)

Fig. 5 Illustrations of, at the 26th layer, (a) the reconstructed image with sparsity via inputting the SVT denoising data into the identity transform; (b) The sole finite difference reconstructed image in combination with the identity transform; (c) Plots of the difference in reconstructed image intensity according to finite difference reconstruction (red solid line) and the finite difference reconstruction with SVT fordenoising (green dash dot line) along 100th row of the reconstructed images.

For further validating the resultant reconstruction related to the current proposed algorithm, we also compute the difference of full images in intensity between the reconstructed images and the original measurement. These difference images are illustrated in Fig. 6 within zoomed in region for clarification. The reconstructed images are in terms of identity transform (IT) with denoising (SVT) and IT itself. After that, we subtract the average of the difference images, then we segment the resultant images with the values of difference in intensity that are large enough to see the error (noisy) pixels. It is clear to observe that the difference image related to IT shown in Fig. 6(b) involves a relatively large number of noisy (error) pixels that are located around the boundary area, which form line shaped region and blur the image edges; while the reconstructed image using SVT for denoising illustrated in Fig. 6(a) shows obviously reduced number of error pixels compared to the reconstruction in Fig. 6(b), where many of error pixels that are shown in Fig. 6(b) have disappeared.

\section{Discussion}

To demonstrate the ability of SVT post-processing scheme for reducing noise pixels and sampling errors, the singular left- and right singular vectors have been plotted in Fig. 2 (b) and (c), respectively, after decomposition of a transform MRI. An under-sampling rate of $94 \%$ has been considered. Figure 2 (b) can be described as follows. Assume a singular value threshold of e.g. $13 \times 260=3380$. Consider 


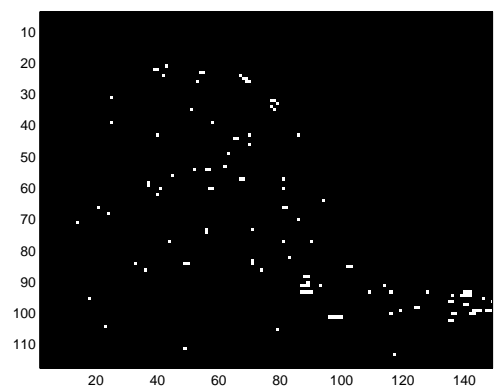

(a)

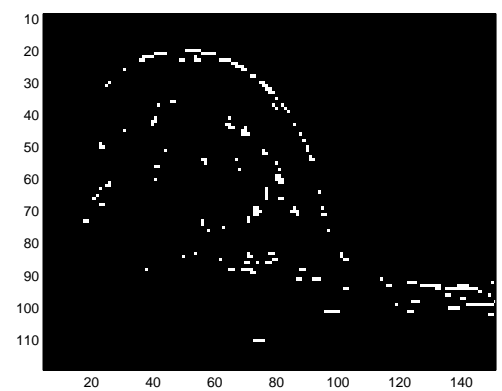

(b)

Fig. 6 Illustrations of resultant experiments at the 26th layer, (a) the difference image segment between the measured MRI and reconstructed image using SVT for denoising. (b) The difference image segment between the measured MRI and transformed image from sampled $k$-space.

the 3380-component left-singular vector $U_{1}$, corresponding to the largest singular value (red line). Also consider the graphs of the left-singular vectors $U_{2}$ (red line) and $U_{3}$ (green line), respectively, corresponding to the next largest singular values. It is seen that the components of the left-singular vectors oscillate moderately strong and decrease comparatively rapidly to negligible values. The inset in Fig. 2(b) shows the largest singular values of the image recovery matrix, where the $y$-axis shows the magnitude of singular values, and the $x$-axis labels the components of singular values.

Figure 2 (c) can be described similarly. Consider the 260-component right-singular vector $V_{1}$, corresponding to the largest singular value (red line). Also consider the graphs of the right-singular vectors $V_{2}$ (red line) and $V_{3}$ (green line), respectively, corresponding to the next largest singular values. The components of the right-singular vectors oscillate stronger than their counterpart leftsingular vectors and decrease less rapidly to smaller values.

Both the left- and right singular vectors of the decomposed image, reproduced in Fig. 2 (a) and (b), respectively, show increased oscillations versus decreased magnitude of SVs. Especially, the right singular vector produced by the largest SV can be approximated to a periodic sine wave with less oscillations. This gives the reason why the larger singular values can be used to achieve good quality reconstruction of an image.

We also conduct FD reconstruction in combination with cosine transform for sparse image presentation, with and without SVT for denoising process. SVT is performed with singular value threshold of $13 \times n(n=260)$. Similar to the FD reconstruction using IT with SVT, the SVT based denoising scheme improves image reconstruction quality compared to sole FD reconstruction. The FD reconstruction with application of DCT takes 962 second to be conducted, the corresponding processing time with SVT is 964 second; and the SVT for denoising costs only 216 seconds. The peak SNR is 12 
with SVT for denoising, which is much improved compared to the peak SNR of 3.5, resulting from the reconstruction without SVT for denoising. Meanwhile, IT in sparse image presentation shows reduced time scale, compared to DCT, in combination with FD for reconstruction. The peak SNR is nearly similar.

Table 2 records the average value among the layers from 27-30 regarding elapsed time using sole SVT, relative error rates and the recovery rates computed via spectrum norm, as well as the number of SVs after image recovery with sampled rates of $94 \%$ and $86 \%$. With the reduced number of largest SVs used to approximately recover the image, there shows improved recovery rate, therefore improved image reconstruction quality. Moreover, there is a tradeoff between relative error rate and image recovery rate. For the case where the sample rate is $94 \%$, the reconstructed image quality under the threshold of $13 \times n$ is close to the sample rate of $86 \%$ with threshold of $34 \times n$. The former shows increased relative error but reduced rank (reduced number of SVs) of a recovered image. Particularly interesting, under the sample rate of $94 \%$, the recovery rate can reach over $98.3 \%$, while the relative error keeps lower than $1.7 \%$, with only 181 out of 260 (70\%) singular values that are calculated. The elapsed time is also shortened with the reduced number of singular values to be calculated. The finite difference reconstruction takes short time to be conducted compared to the FD reconstruction with SVT for denoising. However, the time reduction resulted from FD reconstruction runs the risk of low peak SNR of 2 in combination with identity transform, compared to the peak SNR of 12 when applying SVT to remove noise effect.

Table 2 This is to illustrate, for stable recovery of an image with sampled rates of $94 \%$ and $85 \%$ of original image pixels, in average, the elapsed time, relative error rate (Error ${ }_{\text {rel }}$ ) and recovery rate (Rate rec $)$ computed according to spectrum norm, and the number of SVs after image recovery, with $n \times n=67,600$. We also record the elapsed time of finite difference reconstruction (FD) combining with identity transform (IT), with and without SVT for denoising, as well as relevant peak SNRs.

\begin{tabular}{|c|c|c|c|c|c|c|c|c|}
\hline Rate $_{\text {sample }}$ & \multicolumn{4}{|c|}{$63706 / 67600 \times 100 \%=94 \%$} & \multicolumn{4}{|c|}{$57515 / 67600 \times 100 \%=85 \%$} \\
\hline Threshold & $13 \times n$ & $20 \times n$ & $27 \times n$ & $34 \times n$ & $13 \times n$ & $20 \times n$ & $27 \times n$ & $34 \times n$ \\
\hline Elapsed time & 219 & 209 & 198 & 181 & 208 & 200 & 192 & 180 \\
\hline Error $_{\text {rel }}(\%)$ & 0.4 & 0.8 & 1.2 & 1.7 & 0.3 & 0.6 & 1.1 & 1.5 \\
\hline Rate $_{\text {rec }}(\%)$ & 97.1 & 97.8 & 98.3 & 98.3 & 93.7 & 95.7 & 96.3 & 96.4 \\
\hline Number $_{\text {SVs }}$ & 224 & 206 & 190 & 177 & 216 & 202 & 186 & 174 \\
\hline $\begin{array}{c}\mathrm{SVT}+\mathrm{FD}_{\mathrm{IT}}\left(\mathrm{FD}_{\mathrm{IT}}\right) \\
\text { (elapsed time in FD process) }\end{array}$ & $58(49)$ & $56(43)$ & $52(43)$ & $55(43)$ & $57(43)$ & $53(44)$ & $52(43)$ & $57(43)$ \\
\hline $\begin{array}{c}\text { Peak SNR of } \\
\text { SVT }+ \text { FD }_{\text {IT }}\left(\text { FD }_{\text {IT }}\right)\end{array}$ & $12(2)$ & $12(2)$ & $12(2)$ & $12(2)$ & $10(2)$ & $11(2)$ & $11(2)$ & $11(2)$ \\
\hline
\end{tabular}

Due to the small number of singular values involved in reconstruction, the proposed algorithm for image denoising can be applied in conjunction with a number of methods reported in literature. Three examples have been provided: (i) A kernel-partial-least-squares method can be used to select feature 
sets for improving breast lesion discrimination, and at the same time reducing the computational complexity [18]. (ii) An optimal linear transformation of an image can be conducted to select feature space for activation detection of fMRI, while improving the computational efficiency [19]. (iii) A level-sets method can be utilized to improve the identification of anatomical structures in breast DCE-MRI for motion evaluation [20].

In conclusion, this paper introduces a novel denoising algorithm named SVT for MRI reconstruction after compressive sampling in $k$-space. The current MRI reconstruction via SVT algorithm is a model-based method with application in obtaining a low rank structure for an image matrix. The resultant experiment has demonstrated the ability of SVT to achieve improved quality reconstruction of multi-slice MRIs in terms of compressive sensed $k$-space measurements. SVT is used as a denoising method with a low order decomposed model. Compared to direct use of sparse transform of an image, i.e. DCT, we apply the SVT algorithm onto the FD reconstruction, which shows reduced data storage space for image processing and high quality of image reconstruction performance. The elapsed time of SVT is linearly increased with the increased number of singular values. Our experiments show that, there is a tradeoff between the relative error rate and the relative recovery rate of an image. The peak signal to noise ratio (PSNR) with application of the SVT for denoising is 12, which is comparable to the method suggested by [1], resulting in the peak SNR of only 2 for FD via identity transform and 3 for FD via DCT. It should be pointed out that the method serves as a proof-of-concept only, and motivates future work on further validation with many datasets.

\section{References}

1. M. Lustig, D. Donoho, and J. M. Pauly, "Sparse MRI: The application of compressed sensing for rapid MR imaging," Magnetic Resonance in Medicine 58(6), pp. 1182-1195, 2007.

2. S. Ljunggren, "A simple graphical representation of fourier-based imaging methods," Journal of Magnetic Resonance 54(2), pp. 338-343, 1983.

3. D. Twieg, "The k-trajectory formulation of the NMR imaging process with applications in analysis and synthesis of imaging methods," Medical Physics 10(5), pp. 610-621, 1983.

4. Y. Tsaig and D. Donoho, "Extensions of compressed sensing," Signal Processing 86(3), pp. 533$548,2006$.

5. D. Chralampidis, T. Kasparis, and M. Georgiopoulos, "Classification of noisy signals using fuzzy ARTMAP neural networks," IEEE Transactions on Neural Networks 12(5), pp. 1023-1036, 2001.

6. F. Wichmann, D. Braun, and K. Gegenfurtner, "Phase noise and the classification of natural images," Vision Research 46(8-9), pp. 1520-1529, 2006.

7. N. Wiener, Extrapolation, Interpolation, and Smoothing of Stationary Time Series, Wiley and Sons, New York, USA, 1949.

8. J. F. Cai, E. J. Candès, and Z. Shen, "A singular value thresholding algorithm for matrix completion," SIAM Journal on Optimization 20(4), pp. 1956-1982, 2010. 
9. S. Lingala, H. Yue, E. DiBella, and M. Jacob, "Accelerated dynamic MRI exploiting sparsity and low-rank structure: k-t SLR," IEEE Transactions on Medical Imaging 30(5), pp. 1042-1054, 2011.

10. B. Recht, M. Fazel, and P. A. Parrilo, "Guaranteed minimum-rank solutions of linear matrix equations via nuclear norm minimization," SIAM Review 52(3), pp. 471-501, 2010.

11. S. Osher, Y. Mao, B. Dong, and W. Yin, "Fast linearized Bregman iteration for compressive sensing and sparse denoising," Communications in Mathematical Sciences 8(1), pp. 93-111, 2010.

12. A. Antoulas, Approximation of Large-Scale Dynamical Systems, SIAM, New York, 2005.

13. G. H. Golub and C. F. Van Loan, Matrix Computations, The John Hopkins University Press, Baltimore, MD, USA, 1996.

14. M. K. Schneider and A. Willsky, "Krylov subspace estimation," SIAM Journal on Scientific Computing 22(5), pp. 1840-1864, 2001.

15. Z. Bai, "Krylov subspace techniques for reduced-order modeling of large-scale dynamical systems," Applied Numerical Mathematics 43(1-2), pp. 9-44, 2002.

16. L. Rudin, S. Osher, and E. Fatemi, "Non-linear total variation noise removal algorithm," Physica D 60, pp. 259-268, 1992.

17. Q. Huynh-Thu and M. Ghanbari, "Scope of validity of PSNR in image/video quality assessment," Electronics Letters 44, pp. 800-801, 2008.

18. T. Mu, A. K. Nandi, and R. M. Rangayyan, "Classification of breast masses via nonlinear transformation of features based on a kernel matrix," Medical \& Biological Engineering \& Computing 45(8), pp. 769-780, 2007.

19. F. Sun, D. Morris, and P. Babyn, "The optimal linear transformation-based fMRI feature space analysis," Medical \& Biological Engineering \& Computing 47(11), pp. 1119-1129, 2009.

20. X. X. Yin, B. W.-H. Ng, Q. Yang, A. Pitman, K. Ramamohanarao, and D. Abbott, "Anatomical landmark localization in breast dynamic contrast-enhanced MR imaging," Medical $\mathscr{E}$ Biological Engineering \& Computing 50(1), pp. 91-101, 2012. 


\section{University Library}

\section{- M M N E R VA A gateway to Melbourne's research publications}

Minerva Access is the Institutional Repository of The University of Melbourne

Author/s:

Yin, XX;Ng, BW-H;Ramamohanarao, K;Baghai-Wadji, A;Abbott, D

Title:

Exploiting sparsity and low-rank structure for the recovery of multi-slice breast MRIs with reduced sampling error

Date:

2012-09-01

Citation:

Yin, X. X., Ng, B. W. -H., Ramamohanarao, K., Baghai-Wadji, A. \& Abbott, D. (2012).

Exploiting sparsity and low-rank structure for the recovery of multi-slice breast MRIs with reduced sampling error. MEDICAL \& BIOLOGICAL ENGINEERING \& COMPUTING, 50 (9), pp.991-1000. https://doi.org/10.1007/s11517-012-0920-x.

Persistent Link:

http://hdl.handle.net/11343/283054 